\title{
Analysis and control of ultrafast photon-induced processes
}

DOI: $10.1039 / \mathrm{c1cp90059e}$

This Themed Issue on "Analysis and Control of Ultrafast Photon-Induced Processes" is motivated by several factors. Firstly, it is a timely subject of cutting-edge research involving ultrafast processes which are induced by the impact of short photon pulses. Constantly, the meaning of the word "ultrafast" is changing, since the field of research is developing quickly. Secondly, sources of femtosecond laser pulses are constantly improving, so that from the experimental point of view shorter time scales become accessible to time-resolved experiments. Femtochemistry, as pioneered by A. H. Zewail, is nowadays an almost classical field of research. Present laser sources reach into the attosecond time regime, permitting the probing of electron dynamics by pump-probe experiments. Thirdly, theory provides a deep level of understanding of experimental results and is often ahead of experiments by giving evidence for suitable predictions. This does not only refer to processes induced by ultrashort laser pulses, but also coherent control schemes were developed by theory before experiments were performed. This includes fundamental work by D. J. Tannor and S. A. Rice $(1985)^{1}$ and P. Brumer and M. Shapiro (1986), ${ }^{2}$ who suggested different control schemes, where either the temporal delay of two laser pulses or the phase is used, respectively. Further progress was made by $\mathrm{H}$. Rabitz and co-workers (1988-1992), ${ }^{3}$ who developed optimal control theory, providing closed-loop pulse shaping that is based on genetic algorithms for controlling the dynamics of complex molecular systems. Experimental use of these approaches was made subsequently in pioneering work by the groups of G. Gerber ${ }^{4}$ and Y. Silberberg, ${ }^{5}$ who succeeded in shaping laser pulses for controlling photoinduced reactions.

The Collaborative Research Center 450 has contributed to the topic of this Themed Issue over the last 12 years. The center was established in 1998 in Berlin and has been headed since then by L. Wöste at Freie Universität Berlin. The Collaborative Research Center 450 worked until 2010, when the maximum time period for funding by the German Research Foundation (DFG) was reached. Generous financial support by DFG of Collaborative Research Center 450 is gratefully acknowledged. During 12 years of operation the subject of study underwent a systematic evolution from the analysis of photoinduced reactions towards the control of these processes and transforming them into tools for applications. This required the development of suitable tools for controlling photoinduced reactions, where significant contributions to the progress of this field were made in the past by researchers who worked in this Collaborative Research Center.

The final colloquium of Collaborative Research Center 450 is celebrated together with the Bunsen-Meeting 2011, which is held at Freie Universität Berlin on June 2-4, 2011. There, the main topic of this physical chemistry spring meeting is almost identical to that of this Themed Issue. It is aimed to show recent progress in this field on an international scale, which goes significantly beyond Collaborative Research Center 450. In preparation of the Bunsen-Meeting 2011 several authors from all over the world were requested to contribute to this Themed Issue. We are pleased that finally 16 papers were gathered after the review process, where nine of them cover research performed by Collaborative Research Center 450, documenting exemplarily the progress that was made to this field of research.

We have ordered the contributions starting from ongoing methodological developments, via small and complex molecular systems, reaching finally metallic nanoparticles. Clearly, theoretical work is dominating the contributions and there are new trends of developments visible, which go beyond the scope of Collaborative Research Center 450. These are attosecond electron localization in dissociative ionization (F. Kelkensberg et al.-DOI: 10.1039/ C1CP20058E), waveform control and strong-field induced plasmon oscillations in few-cycle laser fields (I. Znakovskaya et al.-DOI: 10.1039/C0CP02743J-and J. Köhn and T. Fennel-10.1039/ C0CP02344B). Such developments document the perpetual progress in this field indicating that novel ultrafast photoinduced processes and control schemes can be discovered in the future.

We thank the authors for their contributions to this Themed Issue and all reviewers for their skilful work. Finally, it is our pleasure to thank the Editors of PCCP and the Publisher for their constant and helpful support.

\section{References}

1 D. J. Tannor and S. A. Rice, J. Chem. Phys., 1985, 83, 5013 .

2 P. Brumer and M. Shapiro, Chem. Phys. Lett., 1986, 126, 541. 
3 S. H. Shi, A. Woody and H. Rabitz, J. Chem. Phys., 1988, 88, 6870.

M. Strehle and G. Gerber, Science, 1998, 282, 919.

4 A. Assion, T. Baumert, M. Bergt, 5 D. Meshulach and Y. Silberberg, Nature, T. Brixner, B. Kiefer, V. Seyfried,
Vlasta Bonačić-Koutecký, ${ }^{a}$ Nikolaus Ernsting, ${ }^{a}$ Joshua Jortner, Jörn Manz, ${ }^{c}$ Eckart Rühl ${ }^{c}$ and Ludger Wöste ${ }^{c}$

${ }^{a}$ Humboldt University Berlin, Germany ${ }^{b}$ Tel Aviv University, Israel ${ }^{c}$ Freie Universität Berlin, Germany 